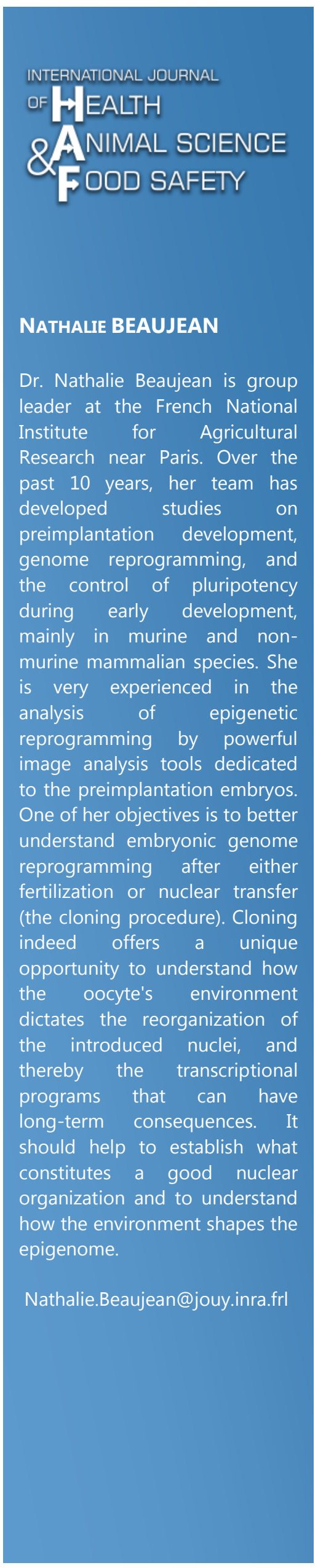

\title{
Histone post-translational modifications in preimplantation mouse embryos and their role in nuclear architecture
}

\author{
Nathalie BEAUJEAN ${ }^{1}$ \\ ${ }^{1}$ INRA, UMR1198 Biologie du Développement et Reproduction, F-78350 Jouy- \\ en-Josas, France
}

\section{MAIN LECTURE}

In mammals, epigenetic markers are globally rearranged after fertilization: while gametes carry special epigenetic signatures and a unique nuclear organization, they attain embryo-specific patterns after fertilization. This "reprogramming" is promoted by the intimate contact between the parental inherited genomes and the oocyte cytoplasm over the first cell cycles of development. Interestingly, histone post-translational modifications (PTMs) are among the factors involved in this reprogramming.

During the last few years, many studies focusing on epigenetic modifications have indeed shown that, immediately after fertilization, different histone PTM profiles create an asymmetry between the two parental genomes, although both parental genomes undergo global hyperacetylation and hypomethylation. Thereafter, histone PTMs reprogramming goes on (Beaujean et al., MRD 2014). It is hypothesized that this PTMs reprogramming is required for the embryonic genome activation (EGA). Recently, we for example put forward the importance of the PRC1 complex that binds $\mathrm{H}_{3} \mathrm{~K}_{2} 7 \mathrm{me} 3$, for proper EGA and development beyond the two-cell stage (Posfai et al., 2012). By the stage of implantation (blastocyst stage) two cell subpopulations forms: an outer layer of epithelial trophectoderm cells (TE) and the inner cell mass (ICM) located eccentrically within the blastocoelic cavity. Remarkably, some histone PTMs have been found to differ between the ICM vs. TE and to correlate with specific gene expression in each of these cell types (Dahl et al., 2010; Vermilyea et al., 2009).

On the other hand, it is well known that diverse parts of the genome have different types of chromatin configuration depending on their function (centromeric and telomeric heterochromatin for instance). Interestingly, the mouse embryo presents a unique organization of the peri-centromeric heterochromatin that locates around the nucleoli. This configuration is rapidly acquired in the maternal pronucleus and more progressively in the paternal one (Martin et al., 2006; Aguirre-Lavin et al., 2012), probably due to the specific epigenetic marks present only in the paternal chromatin. During the 2-cell stage, dissociation of pericentromeric heterochromatin from nucleoli begins, concomitantly with the major phase of embryonic genome activation, although the importance of this remodeling is not yet well understood. Remarkably, it however seems that transcripts generated by pericentromeric satellite repeats are involved in this event and that interference with this phenomenon results in developmental arrest (Probst et al., 2010; Santenard et al., 2010; Fulka \& Langerova 2014).

Altogether, it suggests that histone PTMs may be closely correlated with the formation of a transcriptionally active or repressive state during early embryonic development and that they can modify chromatin organization and nuclear architecture during mouse embryonic development. It should also be mentioned that knock-outs of several histone modification enzymes have underlined the 